EPJ Web of Conferences 81, 02001 (2014)

DOI: 10.1051/epjconf/201481 02001

(C) Owned by the authors, published by EDP Sciences, 2014

\title{
New high precision data on the differential cross sections of the pion-proton elastic scattering
}

I. G. Alekseev1,a, V. A. Andreev², I. G. Bordyuzhin ${ }^{1,3}$, Ye. A. Filimonov², V. V. Golubev², A. B. Gridnev², D. V. Kalinkin ${ }^{1}$, L. I. Koroleva ${ }^{1}$, N. G. Kozlenko², V. S. Kozlov², A. G. Krivshich², B. V. Morozov ${ }^{1}$, V. M. Nesterov ${ }^{1}$, D. V. Novinsky ${ }^{2}$, V. V. Ryltsov ${ }^{1}$, M. Sadler ${ }^{4}$, B. M. Shurygin ${ }^{1}$, A. D. Sulimov' ${ }^{1}$, V. V. Sumachev ${ }^{2}$, D. N. Svirida' ${ }^{1}$, V. I. Tarakanov' ${ }^{2}$, and V. Yu. Trautman ${ }^{2}$ for the EPECUR Collaboration

\footnotetext{
${ }^{1}$ Institute for Theoretical and Experimental Physics, Moscow, 117218, Russia

${ }^{2}$ Petersburg Nuclear Physics Institute, Gatchina, 188300, Russia

${ }^{3}$ National University of Science and Technology "MISiS", Moscow, 119049, Russia

${ }^{4}$ Abilene Christian University, Abilene, Texas, TX 79699-7963, USA
}

\begin{abstract}
The EPECUR collaboration presents new high precision data on the pionproton elastic scattering in the second resonance region. The experiment EPECUR is placed on the universal beam channel of the accelerator ITEP. The setup features $0.1 \%$ beam pion momentum tagging system, $25 \mathrm{~cm}$ long liquid hydrogen target, placed in mylar container and beryllium outer shell, low material wire drift chambers and high performance DAQ. More than 3 billions of triggers have been collected. The data cover pion beam momentum range $0.8-1.3 \mathrm{GeV} / \mathrm{c}$ and $40-120$ degrees center-of-mass scattering angle range for both positive and negative pions. The measured differential cross section has $2 \%$ statistical accuracy in 2 degrees angle and $5 \mathrm{MeV} / \mathrm{c}$ momentum intervals.
\end{abstract}

Spectroscopy of light baryons attracts attention of physicists for already a long time. QCD and quark model are well established for many years. Nevertheless $N$ and $\Delta$-resonance spectra is still not known well (see discussion in [1]). Out of 45 low lying $N$-states predicted only 14 are reasonably well established and 10 are tentative [1]. The main source of established $N$ and $\Delta$-resonances even now is partial wave analyses (PWAs) of pion nucleon elastic scattering [2-4]. The most of the data for these analyses was obtained more than 20 years ago with the experimental technique available at that time. A lot of new high precision data are coming now from photoproduction experiments at the Universities of Bonn, Grenoble, and Mainz, and at the JLab in the US and SPring-8 in Japan. The experiment EPECUR is aimed at providing the new data using pion beam in formation-type experiment. One of the important aims of the experiment is to look for the possible state $N(1685)$, which could be a non-strange member of a baryon anti-decuplet. Evidences for this state were found in photoproduction and Compton scattering off neutron while no signal is seen off proton [5-7]. The state is seen in these experiments as a narrow effect which width is determined by the experimental resolution of about $25 \mathrm{MeV}$. The invariant mass of $\pi p$ system in EPECUR is defined with precision better than $1 \mathrm{MeV}$ by tagging of the incident pion momentum, so width of this state, if seen, can be measured directly.

\footnotetext{
a e-mail: igor.alekseev@itep.ru
} 
EPECUR is a non-magnetic spectrometer with two symmetric arms of wire drift chambers and liquid hydrogen target [8]. Beam particles trajectories are measured in the first focus of the magnetooptic channel with a set of $1 \mathrm{~mm}$-pitch proportional chambers. The whole setup was optimized for a low material count on the particle path. Dedicated DAQ system allows to collect up to $10^{5}$ triggers per accelerator cycle. A soft trigger condition, which required that beam particle did not hit veto counter behind the target, was used during the data taking. Momentum range $810-1340 \mathrm{MeV} / \mathrm{c}$ was scanned in $15 \mathrm{MeV} / \mathrm{c}$ steps with momentum range at fixed beam optics settings about $30 \mathrm{MeV} / \mathrm{c}$. 3.15 billion events were collected with both positive and negative beams. Selection of the elastic events is based on the complanarity and laboratory scattering angles correlation.

The elastic differential cross sections obtained cover $\theta_{\mathrm{CM}}=40-122^{\circ}$ and $P_{\text {beam }}=810-$ $1340 \mathrm{MeV} / \mathrm{c}$ ranges with about $2 \%$ statistical precision in $\approx 9000$ bins of $2^{\circ}$ over $\approx 5 \mathrm{MeV} / \mathrm{c}$. An example of data as a function of the incident pion momentum is shown in figures 1 and 2 for $\pi^{-} p$ and $\pi^{+} p$ correspondingly. Four consecutive angle ranges are shown. Our data is shown by black dots (error bars are not seen because of the dot size). Blue points with error bars show older measurements found in SAID database [4] within $\pm 1^{\circ}$ angle bin. Predictions of the three main PWAs are also drawn: KH80 [2] — dashed red line, CMB [3] — solid black line, and WI08 [4] — dash-dotted blue line. Figures 3 and 4 provide a closer look at the region around invariant mass of $N(1685)\left(P_{\text {beam }}=1024 \mathrm{MeV} / \mathrm{c}\right)$. Some narrow structure can be seen there in many angles (not only ones shown in this paper) in $\pi^{-} p$ scattering, while no significant irregularities are present in $\pi^{+} p$. This can be either some hint of the $N(1685)$ or a manifestation of a casp [9] coming from $K \Sigma$ threshold, though casp can be present in both $\pi^{-} p$ and $\pi^{+} p$ channels.


Figure 1. Differential cross section of $\pi^{-} p$ elastic scattering. 

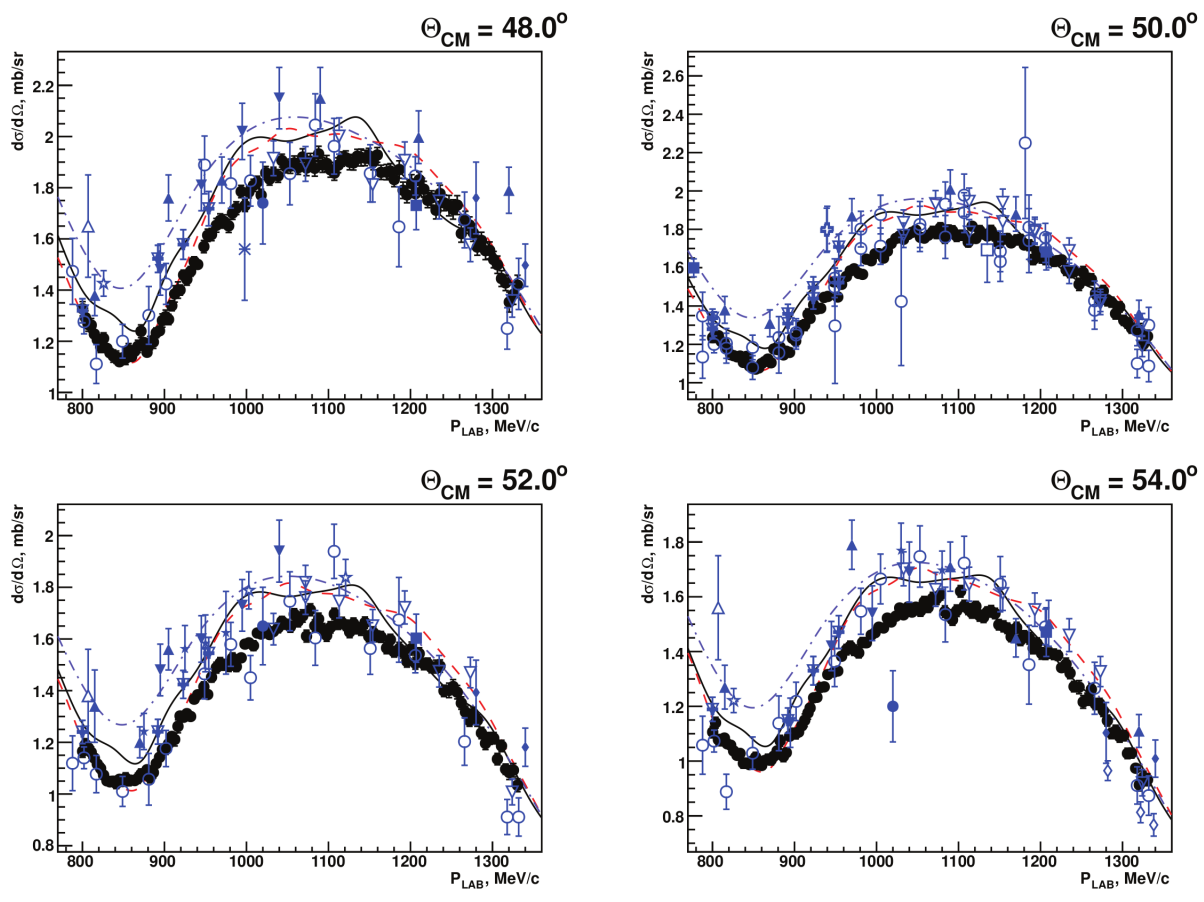

Figure 2. Differential cross section of $\pi^{+} p$ elastic scattering.

The authors wish to acknowledge the excellent support of the accelerator group and operators of ITEP. This work was partially supported by Russian Fund for Basic Research grants 09-02-00998a and 05-02-17005a.

\section{References}

[1] K.A. Olive et al. (Particle Data Group), Chin. Phys. C38, 090001 (2014).

[2] G. Höhler, Pion-Nucleon Scattering, Landolt-Börnstein Vol. I/9b2 (1983), ed. H. Schopper, Springer Verlag.

[3] R.E. Cutkosky et al., Baryon 1980, IV International Conference on Baryon Resonances, Toronto, ed. N. Isgur, p. 19.

[4] R.A. Arndt et al., Phys. Rev. C74, 045205 (2006).

[5] V. Kuznetsov et al., Phys. Rev. C83 022201 (2011); V. Kuznetsov et al., Phys. Lett. B647, 23 (2007).

[6] D. Werthmüller et al., Phys. Rev. Lett. 111, 232001 (2013).

[7] I. Jeaegle et al., Eur. Phys. J. A47, 89 (2011).

[8] I.G. Alekseev et al., Instrum. Exp. Tech. 57, 535 (2014); I.G. Alekseev et al., Nucl. Instrum. Meth. A578, 289 (2007).

[9] A.I. Baz, Zh. Eksp. Teor. Fiz. 33, 923 (1957). 
EPJ Web of Conferences
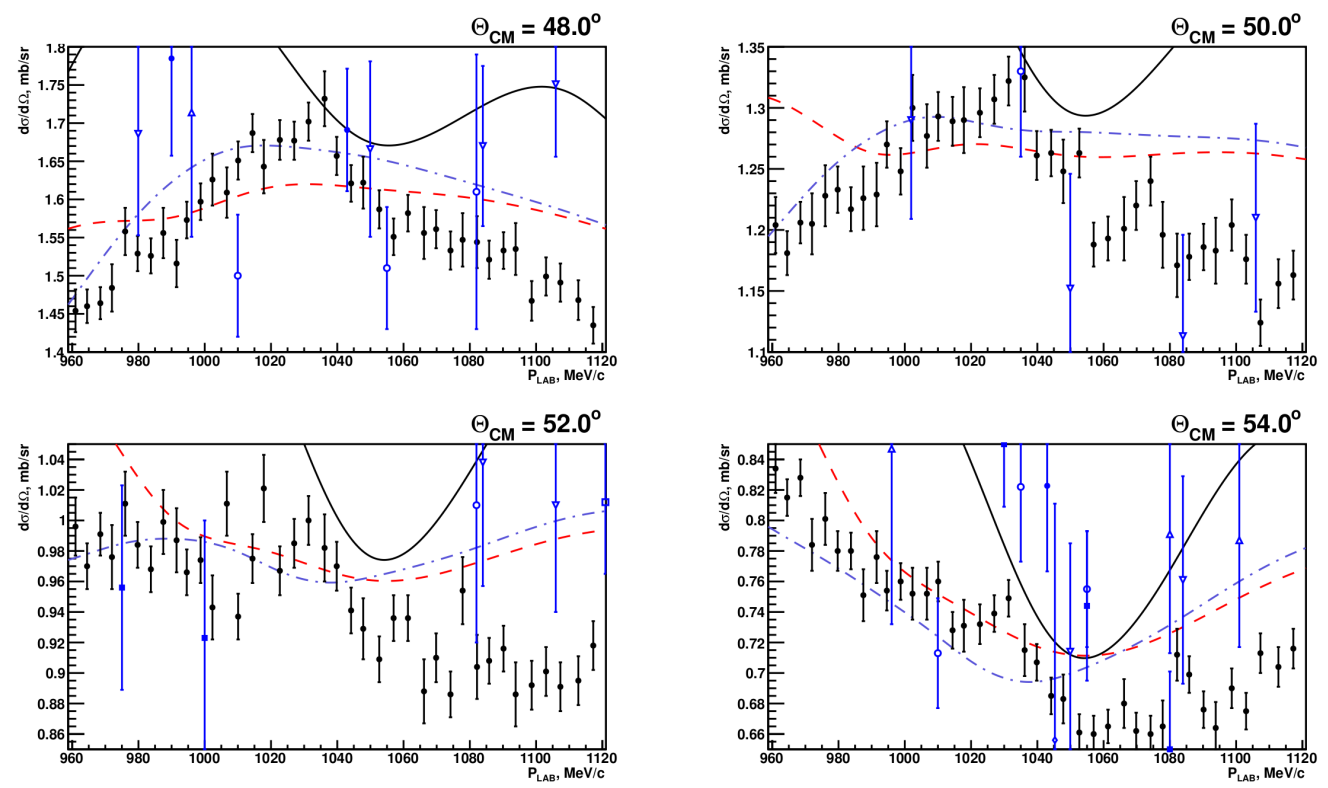

Figure 3. Differential cross section of $\pi^{-} p$ elastic scattering in the region around $N(1685)$.
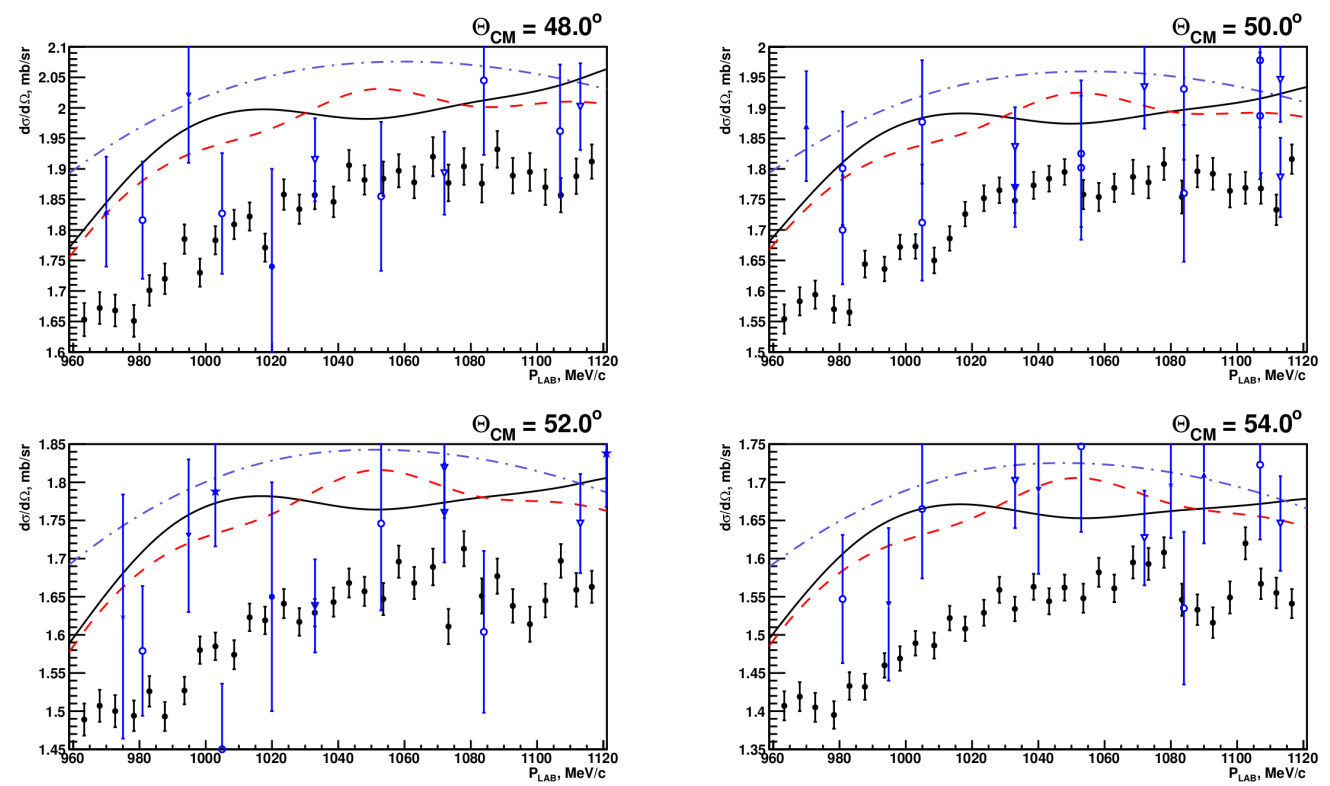

Figure 4. Differential cross section of $\pi^{+} p$ elastic scattering in the region around $N(1685)$. 